\title{
Endemic Plants: From Design to a New Way of Smart Hybrid Nanomaterials for Green Nanomedicine Applications
}

Maroua Ben Haddada ${ }^{1,2}$, Elise Gerometta ${ }^{2}$, Anne Bialecki ${ }^{3}$, Weiling Fu ${ }^{4}$, Yang Zhang ${ }^{4}$, Marc Lamy de La Chapelle ${ }^{4,5}$, Nadia Djaker ${ }^{1}$, Sabrina Pesnel $^{2}$, Anne-Laure Morel ${ }^{2 *}$ and Jolanda Spadavecchia ${ }^{1 *}$

${ }^{1}$ CSPBAT, Laboratoire de Chimie, Structures et Propriétés de Biomateriaux et d'Agents Therapeutiques, Université Paris 13, Sorbonne Paris Cité, Bobigny, France ${ }^{2}$ TORSKAL Nanosciences, 2 Rue Maxime Rivière, 97490 Sainte-Clotilde, La Réunion, France

${ }^{3}$ Laboratoire de Chimie des Substances Naturelles et des Sciences des Aliments, Faculté des Sciences et Technologies Université de, La Réunion, France

${ }^{4}$ Institut des Molécules et Matériaux du Mans, Le Mans Université, Avenue Olivier Messiaen, 72085 Le Mans cedex 9, France

${ }^{5}$ Southwest Hospital, Third Military Medical University, Chongqing, China

\begin{abstract}
In the present study, gold nanoparticles (AuNPs) were successfully developed by green synthesis using a strong antioxidant Quercetin $(\mathrm{Q})$ known as a bioreducing agent, in the presence of Hubertia ambavilla extract plant $(\mathrm{G})$ in order to form hybrid green gold nanoparticles (GQAuNPs). GQAuNPs were characterized by several Spectroscopic techniques (TEM, Raman and UV-Vis spectroscopies) to understand their physicochemical and biological properties. The average particle size was found to be around $20 \mathrm{~nm}$, and corresponding average zeta potential value is $-19 \mathrm{mV}$ suggesting a high stability of GQAuNPs. The large negative potential value could be due to the capping of polyphenolic constituents present in the plant extract. For therapeutic intentions, we propose the important part of GQAuNPs in the hyperthermia treatment of pancreatic cancer through preliminary study based on temperature effect onto colloidal solutions under specific experimental conditions. This study, bringing a green chemical approach, will take up a meaningful, position in the field of nanomedicine, with innovative views for the development of complex- drugs and active targeting in cancer therapy.
\end{abstract}

Keywords: Nanomedicine; Nanoparticles; Hybrid

\section{Introduction}

Previous investigations, in nanomedicine and nanotechnology have established the progress of different nanomaterials with interesting physico-chemical properties for biomedical applications $[1,2]$. Nanoparticles (NPs) can provide many opportunities for the environment and human diseases [3,4]. Toxicological studies showed that small-sized gold NPs (AuNPs) have deleterious effects on the health of humans and living species in the environment [5-7]. In various events, the elevated cytotoxicity of nanoparticles limits their use for medicinal applications [8]. Previously, the grafting of polymers onto gold nanoparticles have led to new and exciting development with a great potential in many fields from chemistry $[9,10]$ to biology $[11]$ and medicine [12].

Recently bio-polymers and phosphonates have applied in nanomedicine [13], thanks to their high chemical stability and low toxicity [14]. Several flavonoids and/or extract plants have been reported in order to conjugate gold nanoparticle surfaces by chemisorption or physisorption $[15,16]$.

AL Morel et al. have reported a green chemistry approach to synthesize AuNPs by the bio-reduction of the $\mathrm{AuCl}_{4}^{-}$ions using endemic and plants originated from Reunion Island and registered in the french pharmacopeia Hubertia ambavilla and Hypericum lanceolatum extracts, in two different methods in substitution to citrate mostly used in common methodology [17]. One of this patented methods is reverse synthesis leading to biocompatible \& stable, $20 \mathrm{~nm}$-sized spherical shape nanoparticles. As we are limited by the depth penetration of the laser, we use apply this technology to treat skin cancer. To date, few studies explain the formation of gold nanoparticles before or concomitantly to the nucleation step. Some authors have studied the mechanism of hybrid nanoparticles and chelation process with drugs on the growth process $[9,18]$.

Thus the grafting of bio-ligands to the nanoparticle surface drew a lot of attention for the development of highly functional hybrid materials
[19]. More recently, flavonoids have been successfully investigated in photothermal therapy (PTT) [20].

PTT, clinically termed as hyperthermia, is a minimal-invasive therapeutic strategy for the destruction of cancerous cells as well as bacteria. It is based on the conversion of photon energy into cytotoxic heat upon near infrared (NIR) laser irradiation [21,22].

Quercetin (3,30,40,5,7-pentahydroxyflavone) Figure S1 is a plant-derived aglycone flavonoid which has shown one of the highest antioxidative potential among flavonoids and different biological effects [23]. Due to this high antioxidative property, quercetin imparts many crucial biological properties like antitumor, anti-inflammatory and antimicrobial activities alone $[23,24]$ and can be encapsulated in nanoparticles as PLGA [25]. We report herein an original method to obtain controlled small nanoparticles coated with an antioxidative flavonoids. This study shows the role of flavonoids as reducing and capping agents. Thus, we presents an innovative manner to screen new PTT agents using green chemistry, considering two main criteria: the size and the in vitro photothermal activity. The aim of this article

${ }^{*}$ Corresponding author: Jolanda Spadavecchia, CNRS, UMR 7244, CSPBAT, Laboratoire de Chimie, Structures et Propriétés de Biomateriaux et d'Agents Therapeutiques, Université Paris 13, Sorbonne Paris Cité, Bobigny, France, Tel: +33(0)148388526; E-mail : jolanda.spadavecchia@univ-paris13.fr

Anne-Laure Morel, TORSKAL Nanosciences, 2 rue Maxime Rivière, 97490 Sainte-Clotilde, La Réunion, France, Tel: +262(0)262938831; E-mail: annelaure.morel@torskal.fr

Received: October 17, 2018; Accepted: October 26, 2018; Published: October 29 2018

Citation: Haddada MB, Gerometta E, Bialecki A, Fu W, Zhang Y, et al. (2018) Endemic Plants: From Design to a New Way of Smart Hybrid Nanomaterials for Green Nanomedicine Applications. J Nanomed Nanotechnol 9: 518. doi: 10.4172/2157-7439.1000518

Copyright: (c) 2018 Haddada MB, et al. This is an open-access article distributed under the terms of the Creative Commons Attribution License, which permits unrestricted use, distribution, and reproduction in any medium, provided the original author and source are credited. 
is twofold: first, we implemented a previous chemical protocol to realize novel green nanoparticles (GQAuNPs) by a one-step procedure from gold-extract plant complex. Second, we investigated the PTT activity of GQAuNPs, before and after internalization in pancreatic adenocarcinoma cells (MIA PaCa-2) in order to evaluate their capacity for ablation of cancer cells. As PTT uses laser irradiation, even if it is a near infrared laser the thickness of tissue that can be treated is limited, so this treatment is particularly well adapted to superficial cancers like melanoma or basal cell carcinoma. So we performed a first in vivo study on a murine melanoma model.

\section{Experimental}

\section{Reagents and chemicals}

Analytical grade tetrachloroaurate salt $\left(\mathrm{HAuCl}_{4}, 99.98 \%\right)$, Quercetin (Q), D-Glucose (G), folic acid (F), Phosphate buffer solution (PBS), were purchased from Sigma-Aldrich, USA.

The methodology used was described previously [17].

\section{Synthesis of green gold nanoparticles (GQAuNPs)}

$5 \mathrm{~mL}$ of $\mathrm{HAuCl}_{4}{ }^{*} 3 \mathrm{H}_{2} \mathrm{O}(0.94 \mathrm{mM})$ was heated to $50^{\circ} \mathrm{C} .1 \mathrm{~mL}$ of TSK1 $(0.96 \mathrm{mg} / \mathrm{mL})$ and $20 \mu \mathrm{L}$ quercetin (Q) $(0.5 \mathrm{mg} / \mathrm{mL})$ were rapidly introduced into the flask with drastic stirring, respectively. The mixtures were continuously heated for a 5 min till a ruby-red color appeared. The flask was cooled for two minutes in an ice bath to stop the reaction.

\section{Characterization of synthesized GQAuNPs}

After synthesis, the nanoparticles were subjected to characterization as described previously [17].

\section{Stability of GQAuNPs}

For stability studies, GAuNPs were dispersed in PBS (0.1 M; pH 5.5), and absorption spectra collected over 3 months (Figure S2).

\section{Photothermal property}

To evaluate whether the fabricated AuNPs were able to absorb the 808-nm NIR laser and generate heat for photothermal therapy, the concentration was adjusted to OD (plasmon) $=1$ in water. After a session of NIR laser irradiation $(3 \mathrm{~W}, 808 \mathrm{~nm})$, the temperature curve of the $1 \mathrm{~mL}$ AuNPs was measured using the thermocouple every $30 \mathrm{~s}$ for $10 \mathrm{~min}$.

\section{In vitro biological activity of GQAuNPs}

Cell culture: MIA PaCa-2 cells, a human pancreatic carcinoma cell line, were obtained from the AmericanTissue Culture Collection (ATCC) and were cultured as described previously [14].

Evaluation of GQAuNPs cytotoxicity: The toxicity was measured by comparing the cell proliferation rates with and without AuNP for 24 h. Cells were seeded at a density of $0.25 \times 10^{6}$ cells $/ \mathrm{mL}$ in 48 -well plates to make the cells adherent and proliferating. After $24 \mathrm{~h}$ of incubation, $125 \mu \mathrm{L}$ of the culture medium was replaced with AuNPs (Q1, Q2, Q3, Q4 and QTSK1) or free Quercetin at the same final concentration in AuNPs, and the cells were further incubated for $24 \mathrm{~h}$. Control cells were used without AuNPs/Quercetin treatment. The number of cells was determined by counting with a Coulter Counter Z2 (Beckman) and each experiment was performed in triplets for each sample. The average of all of the experiments has been shown as cell-viability percentage in comparison with the control experiment, while gold untreated controls were considered as $100 \%$ viable.
Cell internalization procedure: To facilitate the uptake of nanoparticles into the cells, and to enhance their biocompatibility, the synthetized gold nanoparticles were conjugated to folic acid (F) and glucose $(G)$. First, the synthesized gold nanoparticles were centrifuged for $30 \mathrm{~min}$ at a speed of $11,000 \mathrm{rpm}$. The precipitate was resuspended in an aqueous solution containing G. The solution was then incubated at room temperature with continuous stirring. After $1 \mathrm{~h}$, the solution was centrifuged and the precipitate was collected and resuspended in an aqueous solution containing $\mathrm{F}$ and left for $1 \mathrm{~h}$. The mixture was then centrifuged and redispersed in water containing $9 \% \mathrm{NaCl}$. The obtained AuNPs are called GQAuNPs@G@F.

MIA PaCa-2 cells were seeded into a Lab-Tek ${ }^{\otimes} I$ Chamber Slide $^{\mathrm{Tt}}$ System and maintained in DMEM supplemented with $10 \%$ FBS for $24 \mathrm{~h}$, then washed three times with PBS and incubated with the gold nanoparticles solution. After $24 \mathrm{~h}$, the cells were rinsed with PBS and fixed with $4 \%$ paraformaldehyde at room temperature for 15 minutes and washed with PBS.

Optical imaging: The optical images of the cells have been made with the optical microscope of an Xplora Raman microspectrometer (Horiba Scientifics) in bright and dark fields. 20x and 80x objectives have been used with numerical apertures of 0.25 and 0.75 respectively.

Photothermia experiments: A $660 \mathrm{~nm}$ laser diode is focused on the cells and the gold nanoparticles using a 80x objective (Numerical aperture $=0.75$ ) using the optical microscope of an Xplora Raman microspectrometer (Horiba Scientifics) with a laser irradiation of 2.2 $\mathrm{mW} / \mu \mathrm{m}^{2}$.

\section{In vivo evaluation of GQAuNPs}

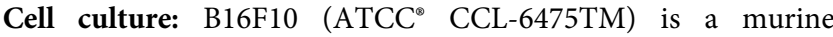
melanoma cell line from a C57Bl/6J mouse. Cell line was cultured in MEM medium supplemented with FBS (10\%) and L-glutamine (2 $\mathrm{mM}$ ). Cells are maintained in an incubator at $37^{\circ} \mathrm{C}$ with $5 \% \mathrm{CO}_{2}$.

Animals and tumors: $\mathrm{BALB} / \mathrm{c}$ nude male mice, 5 weeks old were used. Animals were housed in plastic cages inside a controlled ventilated rack with free access to water and food. All experiments were performed in accordance with national animal care guidelines (EC directive 86/609/CEE, French decree ${ }^{\circ} 87-848$ ). Tumor xenografts were achieved by subcutaneous injection of tumor cells suspension $\left(2.5 \times 10^{5}\right.$ cells in $200 \mu \mathrm{l}$ PBS $)$ into the right flank. To follow tumor growth, tumor volume $\left(\mathrm{V}\right.$ in $\left.\mathrm{mm}^{3}\right)$ was measured with a cliper and was calculated as $\mathrm{V}=$ (length $\mathrm{x}$ width $\mathrm{x}$ thickness) $/ 2$.

Photothermia treatment: When tumors reached a volume of 200 $\pm 50 \mathrm{~mm}^{3}$ (D0), mice were randomly assigned into 3 groups (5 mice per group). In the CTRL group, mice were not treated; in the Laser group, PBS $(100 \mu \mathrm{L})$ was injected into the tumor then the mice received photothermia treatment; in the AuNPs group, GQAuNPs@G@F (100 $\mu \mathrm{L}$ ) was injected into the tumor then the mice received photothermia treatment. Tumor treatment was performed only 1 hour after injection of PBS or AuNPs. During all treatment procedure mice were anesthetized and a $808 \mathrm{~nm}$ laser diode was positioned above the tumor. Each tumor was irradiated $20 \mathrm{~min}$ with a power density of $1.5 \mathrm{~W} / \mathrm{cm}^{2}$.

Evaluation of antitumor activity: Treatment efficacy was assessed in terms of the compound's effects on tumor volume for PTT-treated mice relative to control vehicle-treated mice. Two evaluation criteria were used in parallel: (i) Growth inhibition, calculated as the ratio of the median tumor volume of NP-treated versus control groups : T/C, \% $=($ median tumor volume of NP-treated group on day X / median tumor 
Citation: Haddada MB, Gerometta E, Bialecki A, Fu W, Zhang Y, et al. (2018) Endemic Plants: From Design to a New Way of Smart Hybrid Nanomaterials for Green Nanomedicine Applications. J Nanomed Nanotechnol 9: 518. doi: 10.4172/2157-7439.1000518

volume of control group on day X) x 100, the optimal value, being the minimal $\mathrm{T} / \mathrm{C}$ ratio which reflects the maximal tumor growth inhibition achieved; (ii) Relative area under the tumor growth curve, rAUC (\%), representative of the tumor growth curve as a whole, reflects the overall effect of a test compound over time. rAUC $=[$ (area under the tumor volume growth curve of the treated group / median area under the tumor volume growth curve of the control group) $\mathrm{x}$ 100]. The more active the compound, the lower the rAUC value.

\section{Results and Discussion}

\section{Synthesis mechanism of GQAuNPs}

Previously A. Morel et al. have developed a green methodology in order to obtain a nano-therapeutic agent based on a gold-plant complex [17] (FR 16/50520, PCT/FR2017/050131)). The reverse synthesis method is a universal approach to obtain green nanoagents for medical applications. This could be considered as nanoplatforms allowing diverse functionalizations. Chemico-physical characterizations of flavonoids demonstrated their impact on the design (shape and size) on the synthesis of gold nanoparticles (data not published). We assume that the nature of flavonoids, thus the position of functional groups $(-\mathrm{OH})$ and the change of biomolecules conformation during the formation of gold-nanostructure by complexation, have a wide influence in its plasmonic therapeutic activity. Previous studies reported that the nature of the polyphenols used for the bioreduction of gold can affect the antioxidant activity $[26,27]$.

Other authors have studied the mechanism of gold-drug-complex and competition phenomena with various ligands on the growth process [14,28-31].

It was established that natural flavonoids such as quercetin, daizeol and puerarin, can act as reductants for the enlargement of gold nanoparticles implicated in optical detection [32,33]. In our previous work, the plant extract produced in Reunion Island obtained from Hubertia ambavilla (TSK1) was composed of flavonoids containing hyperoside, the main flavonoid (data not published) and in a small contribution, quercetin.

In this present study, we report a novel chemical protocol developed to better understand the chemical mechanism implicated in the formation of green gold nanoparticles using well known and widely used quercetin. This will bring additional properties to improve the therapeutic efficacy of green gold nanoparticles through formation of stable complexes of flavonoid plant extract and $\mathrm{Au}$ (III) as building blocks of gold nanoparticles.

The major difference with previously reported synthetic procedures is that both TSK1 (extract plant) and quercetin (Q) reacts to the stabilization of AuNPs via chelation between ketone and hydroxyl groups with chloride auric ions. The mechanism of green synthesis of gold nanoparticles using catechine has been described [34]. The reducing agent seems to require $-\mathrm{OH}$ groups present in the catechin moieties to reduce gold and chelate Au. Catechin molecules act as bidentate ligand. The protonation and deprotonation of the $\mathrm{B}$ ring plays a crucial role in reducing $\mathrm{Au}$ (III) and the radical scavenging activity of catechin.

In this report, we suppose a necessary formation of a stable complex between $\mathrm{Au}^{3+}$ and flavonoids by chelation before the reduction. In our case, the formation of gold NPs from $\mathrm{AuCl}_{4}^{-}$includes these main steps (Scheme 1):

(1) Complexation of plant extract (TSK1)- $\mathrm{AuCl}_{4}{ }^{-}$and generation of gold clusters (Hybrid Complex 1).
(2) Initial reduction of TSK1-metal that adsorbs onto Au III to form $\mathrm{Au}$ II

(3) Staking process of quercetin (Hybrid complex 2):

(4) Final reduction of metal ions to form gold particles stabilized by molecules of plant extract.

In the first step, a mixture of extract plants (TSK1) were added in $\mathrm{HAuCl}_{4}$ aqueous solution to complex with it. Thus, the positively charged of flavonoid in the water solutions shows strong electrostatic interaction with negatively charged $\mathrm{AuCl}_{4}^{-}$ions and they form a complex flavonoid- $\mathrm{AuCl}_{4}^{-}$called Hybrid Complex 1 (Scheme 1). The addition of quercetin (Q) on the hybrid complex 1, stabilizes it through electrostatic interaction between carbonyl and amino groups (Hybrid complex 2) improving the kinetics of reduction by complexation of $\mathrm{Au}$ ions [35], controlling the growth process of final green nanoparticles (GQAuNPs) under control of temperature. All products were extensively characterized by UV-Vis absorption spectroscopy, TEM and Raman Spectroscopy.

\section{Drug design of GQAuNPs and comparative physico-chemical characterizations}

We test the efficiency of quercetin, a well-known naturally occurring hydroxyflavone for the synthesis of gold nanoparticles and compared the results with the performance of the plant extracts. In order to evaluate the quercetin $(\mathrm{Q})$ as a reducing agent to form gold nanoparticles (AuNPs), different concentrations of $\mathrm{Q}$ were added to the chloroauric acid $\left(\mathrm{HAuCl}_{4} \cdot 3 \mathrm{H}_{2} \mathrm{O}\right)$ following the same synthesis conditions as QTSK1.

Table 1 presents the different samples with the corresponding Q concentrations.

$\mathrm{Q}$ is sparingly soluble in water and soluble in organic solvents such as ethanol, DMSO, and DMF which are forbidden for green synthesis of NPs. At the chosen concentrations, quercetin remains in suspension which must be passed to the sonicator before addition to the gold salt solution.

The appearance of a purple color following mixing of $\mathrm{Q}$ with $\mathrm{HAuCl}_{4}$ solution indicated the formation of AuNPs (Figures S3 and S4). The resulting colloidal solutions were examined by UV-vis

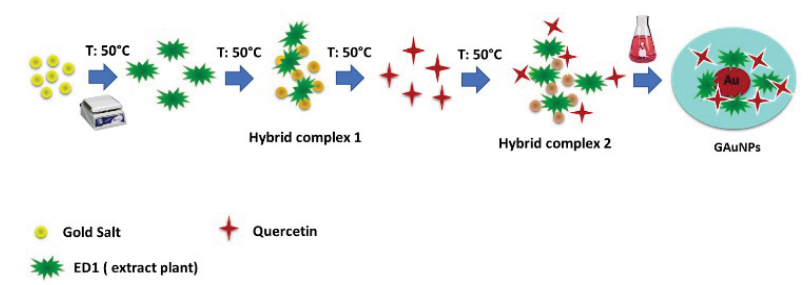

Scheme 1: Schematic representation of GQAuNPs synthesis via a 4-step process (Please note that drawings are not in scale and are not intended to be representative of the full samples composition).

\begin{tabular}{|c|c|c|}
\hline Sample & [Quercetin $_{\text {final }}(\boldsymbol{\mu g} / \mathbf{m L})$ & $\boldsymbol{\Lambda}_{\text {max }}(\mathbf{n m})$ \\
\hline Q1 & $333.34 \mu \mathrm{g} / \mathrm{mL}$ & 558 \\
\hline Q2 & $166.67 \mu \mathrm{g} / \mathrm{mL}$ & 562 \\
\hline Q3 & $83.34 \mu \mathrm{g} / \mathrm{mL}$ & 542 \\
\hline Q4 & $41.67 \mu \mathrm{g} / \mathrm{mL}$ & $570-600$ \\
\hline Q5 & $16.67 \mu \mathrm{g} / \mathrm{mL}$ & - \\
\hline QTSK1 & $1.67 \mu \mathrm{g} / \mathrm{mL}$ & 535 \\
\hline \multicolumn{3}{|c}{ Table 1: Samples characteristics. } \\
\hline
\end{tabular}


Citation: Haddada MB, Gerometta E, Bialecki A, Fu W, Zhang Y, et al. (2018) Endemic Plants: From Design to a New Way of Smart Hybrid Nanomaterials for Green Nanomedicine Applications. J Nanomed Nanotechnol 9: 518. doi: 10.4172/2157-7439.1000518

spectroscopy. Figure S5 presents typical spectra that were obtained using the 5 concentrations and compared to candidate QTSK1. Plasmon resonance peaks for the obtained solutions were recorded as an indicator for NPs formation.

The minimum needed concentration of $\mathrm{Q}$ to form NPs is between 20 and $40 \mu \mathrm{g} / \mathrm{mL}$. For the synthesis of QTSK1, only $1.67 \mu \mathrm{g} / \mathrm{mL}$ was added which confirms that the formation of QTSK1 is mainly due to the reduction of the gold salt by the totum of flavonoid. Hubertia ambavilla contains mainly hyperoside. Thus, we made the hypothesis of a synergetic interaction between hyperoside and quercetin during QTSK1 synthesis. This could improve the reduction of Au (III) in $\mathrm{Au}(0)$, nucleation, aggregation, growth and stabilization of AuNPs. The synthetized NPs (Q1-Q5) were instable compared to QTSK1 and formed aggregates deposed in the bottom of the tube after only $5 \mathrm{~h}$ cooling at room temperature (Figure S4 B). Therefore, they are not suitable candidates for imaging and drug-delivery applications in the human body due to the problem of cells internalization. The plant extract are more relevant protecting agent than Q. It is hydrosoluble, it adsorbs onto the surface of the newly formed nanoparticles (NPs) to prevent further growth and particle agglomeration. The heat generation property of the obtained NPs was also evaluated in water. Figures 1 and 2 shows a plot of the temperature rise in the NPs solution versus time. QTSK1 shows high thermal properties compared to the sample prepared with the only $\mathrm{Q}\left(60 \mathrm{Vs} 35^{\circ} \mathrm{C}\right)$.

\section{Physico-chemical characterization of selected GQAuNPs}

Phenols are analogs in the chemical structure and present an aromatic ring bearing one or more -OH groups [36].

Polyphenols, in particular flavonoids are considered to be ubiquitous in plants. Flavonoids are the largest group of phenolic plants. They consist in two aromatic rings $\mathrm{A}$ and $\mathrm{B}$ joined by a three carbon bridge (ring $\mathrm{C}$ ) as seen in Figure S1. Hydroxyl functions and ketone groups play a key role during complexation and reduction of gold ions. The appearance of a red color following mixing of the Hubertia ambavilla extract with $\mathrm{HAuCl}_{4}$ solution indicated the formation of GQAuNPs. The gold nanoparticles were found to be stable even after repeated centrifugation and washing. Thus, phytochemicals substances presents in plant extract are presumably responsible for the creation of coating on gold nanoparticles and thus, rendering the nanoparticles stable against agglomeration. Plant extract possessed the dual role of reducing gold ions and providing stabilization effects to the formed nanoparticles. Gold nanoparticles produced by this green synthesis did not require any external chemicals agents for the reduction and stabilization of the nanoparticle.

$\mathrm{UV}$-vis spectroscopy is one of the most important techniques to determine and evaluate the formation and stability of metal nanoparticles in aqueous solution.

Figure $3 \mathrm{~A}$ shows a typical spectrum that were obtained after centrifugation. The absorbance was observed around $\lambda_{\max }=536 \mathrm{~nm}$ due to the excitation of the surface plasmon vibrations in the GQAuNPs. This demonstrates the complex formation by chelation of $\mathrm{Au}$ (III) and quercetine. It is further confirmed by other characterizations that this peak indicates the formation of monodispersed spherical shape GQAuNPs.

The morphology and size of the synthesized nanoparticles were determined by TEM images showed in Figure 3B. Images reveal that the GQAuNPs are predominantly spherical in shape and are not in physical contact with each other. Lower magnification image reveals

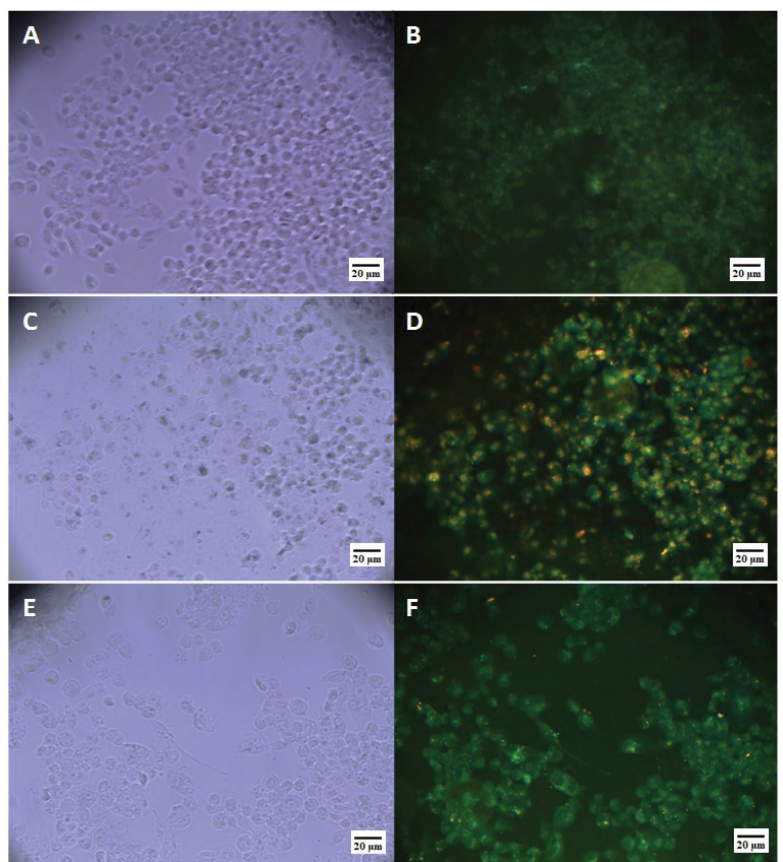

Figure 1: Bright field $(A, C, E)$ and dark field $(B, D, F)$ images $(20 x, 0.25 \mathrm{NA}$ objective) of MIA PaCa-2 cell lines untreated $(A, B)$ and treated with initial concentration (C,D) et 5 time diluted concentration (E,F) of GQAuNPs@G@F. The same sample region is monitored above and below.

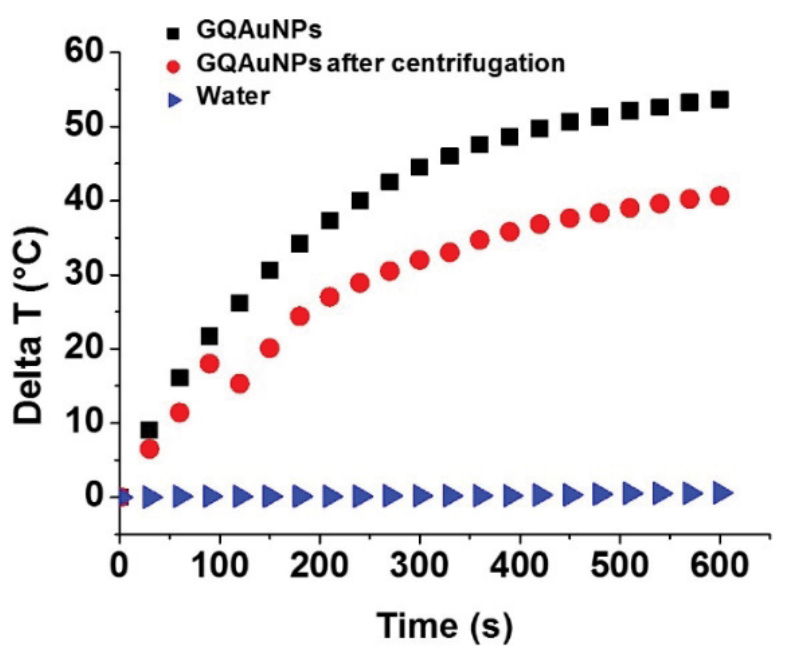

Figure 2: Time-dependent temperature elevation of GQAuNPs before (black line) and after (red line) centrifugation in aqueous solution under $808 \mathrm{~nm}$ laser $\left(1 \mathrm{~W} . \mathrm{cm}^{-2}\right)$.

that the nanoparticles are embedded in a dense matrix which may be the organic stabilizing components of Hubertia ambavilla extract. We proceeded to further sequential centrifugation steps to reduce the density of the matrix leading individual nanoparticles. The average diameter of these AuNPs is $20 \pm 3.8 \mathrm{~nm}$, which is determined by selecting 20 particles randomly from a TEM photograph as shown in Figure 3B.

The zeta potential of AuNPs is commonly used to characterize the surface charge property of nanoparticles. It reflects the electrical 
Citation: Haddada MB, Gerometta E, Bialecki A, Fu W, Zhang Y, et al. (2018) Endemic Plants: From Design to a New Way of Smart Hybrid Nanomaterials for Green Nanomedicine Applications. J Nanomed Nanotechnol 9: 518. doi: 10.4172/2157-7439.1000518

A)

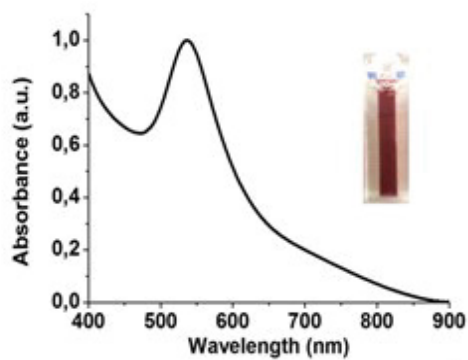

c)

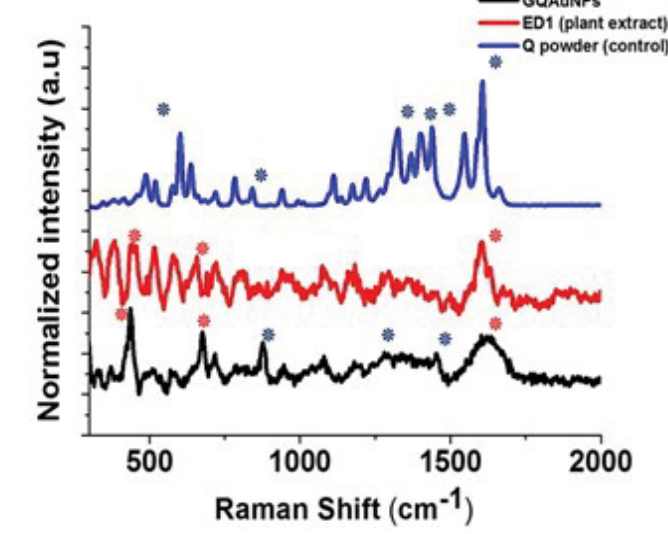

B)

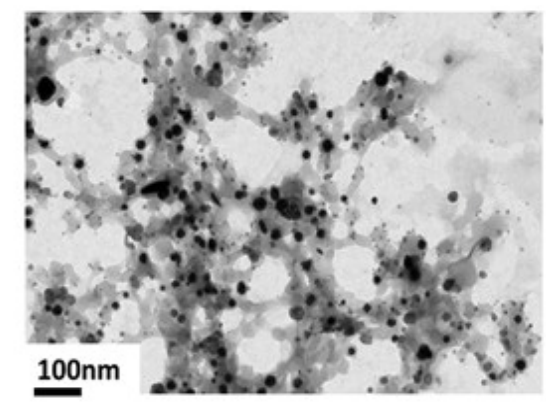

D)

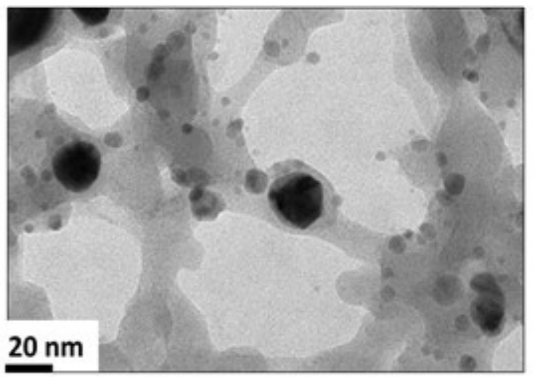

Figure 3: (A) Normalized UV-Vis absorption, (B) TEM and High TEM magnification (D) images, (C) normalized Raman spectra of the selected GQAuNPs (QTSK1) compared to TSK1 extract plant and free Quercetin (Q) as control. (B, D) Scale bars: $100 \mathrm{~nm}$ and $20 \mathrm{~nm}$. (C) Experimental conditions: $\lambda_{\text {exc }}=785 \mathrm{~nm}$; laser power $22 \mathrm{~mW}$; accumulation time $100 \mathrm{~s}$.

potential of particles and is influenced by the composition of the particle and the medium in which it is dispersed. This is a very important indicator in the evaluation of the stability of colloidal systems and their renal clearance for biological application. Hak Soo Choi and al [37] demonstrated that the charge of the coating had a huge effect on the adsorption of serum proteins as well as the hydrodynamic diameter. Zwitterionic or neutral coatings prevented adsorption of serum proteins, which otherwise was associated with an increase in hydrodynamic diameter $>15 \mathrm{~nm}$ and prevented renal excretion. The biosynthesized AuNPs was further demonstrated and confirmed by the characteristic peaks observed in Size distribution and zeta potential curve (Figure S3), which indicate that the average of the diameter was in the range is $35 \mathrm{~nm}$ and corresponding average zeta potential value is $-19 \mathrm{mV}$ suggesting a high stability of GQAuNPs. The large negative potential value could be due to the capping of polyphenolic constituents present in the plant extract. The difference in size of particles observed by DLS when compared to TEM is the fact that the measured size also includes the bio-organic compounds enveloping the core of the GQAuNPs as seen in TEM. Thestability of GQAuNPs in solution was monitored by the Localized Surface Plasmon (LSP) band at $536 \mathrm{~nm}$. Analysis was carried out at and electrolytic conditions $(\mathrm{pH}$ 5.5). The synthesized GQAuNPs did show an almost negligible change in the LSP band position over a period of three months (Figure S2).

We have also recorded the Raman spectrum of the synthesized GQAuNPs and compared it with the Hubertia ambavilla extract (TSK1) and quercetin (Q) as control (1). The main goal was to identify similarity between extract and colloidal solution that indicated that the same compounds existed in both media. Raman spectral peak intensities from the plant extract were observed signifying that the Raman-reporter molecules from the extract were retained on the
AuNPs during the synthesis process. The SERS spectrum in Figure 3C demonstrates the low wavenumber region (i.e. at $483,438 / 431,526 \mathrm{~cm}$ ${ }^{1}$ ) which are related to a possible oligomerization of quercetin (Q) [38]. This confirms the third step of staking process of quercetin (hybrid complex) in the synthesis of GQAuNPs.

As previously described [17], the overlapping peak of the functional group of the Hubertia ambavilla and quercetin present on the AuNPs corroborates also results in that chemical compounds from the plant extract had been coated on the surface of AuNPs and formed chemical bond with AuNPs. Concerning the photothermal effect studied of GQAuNPs, we says that the phenomenon occurs when a metallic nanoparticles converts the absorbed photon onto heat [39] depending on nanoparticle nature, shape and size, the treated cancer cells [40], drug delivery and photodynamic therapy for their ability to select drive temperature-sensitive chemical reactions [41]. In order to realize a good PTT nanovector, a large number of conditions should be accomplished, including size and shape for a NIR region illumination, but also a low cytotoxicity to healthy tissues [42]. Recently, druggold nanovectors have demonstrated excellent properties that can be applied to areas such as drug delivery, cell separation and hyperthermia $[29,43]$.

In our study, the heat generation property of the synthesized GQAuNPs was first determined in water solution. Figure 2 shows a plot of the temperature rise $(\Delta \mathrm{T})$ in the GQAuNPs solution versus time. $\Delta \mathrm{T}$ reached almost $45^{\circ} \mathrm{C}$ after $5 \mathrm{~min}$ of laser irradiation before the centrifugation of nanoparticles which consisted in eliminating the excess of polyphenolic compounds at the surface of nanoparticles. After centrifugation, the hydrodynamic diameter of the GQAuNPs is reduced, confirming the release of an excessive coating probably bound by $\mathrm{H}$ bonds, pi-pi stacking interactions through aromatic phenols 
Citation: Haddada MB, Gerometta E, Bialecki A, Fu W, Zhang Y, et al. (2018) Endemic Plants: From Design to a New Way of Smart Hybrid Nanomaterials for Green Nanomedicine Applications. J Nanomed Nanotechnol 9: 518. doi: 10.4172/2157-7439.1000518

Page 6 of 9

rings. The temperature change in the control medium was less than $2^{\circ} \mathrm{C}$ during NIR irradiation. These demonstrated that the $808 \mathrm{~nm}$ NIR laser can induce SPR on the NPs and generate heat, increasing the temperature of the solutions. The results show a decrease of $10^{\circ} \mathrm{C}$ after centrifugation compared to the initial NP.

The thermal destruction only requires an increase of about $7^{\circ} \mathrm{C}$ provoking the irreversible destruction of malignant cells. New and further results will later highlight the biological mechanism of cell destruction (necrosis vs. apoptosis) during PTT.

\section{In vitro biological activity of GQAuNPs}

The aim of this study is to confirm the green-nanoparticle candidates for the in vitro and in vivo tests. The cell viability (\%) following $24 \mathrm{~h}$ exposure to the prepared GQAuNPs compared to free quercetin at the same concentration (Table 1) was evaluated (Figures S6 and S7). It was found that free $\mathrm{Q}$ and GQAuNPs decreased the cell viability. GQAuNPs were more cytotoxic than free quercetin, suggesting that the degree of cytotoxicity for quercetin was increased by conjugation of quercetin to the GQAuNPs. Kumar et al. [44] have previously showed the significant induction of cytotoxicity of quercetin conjugated $\mathrm{Fe}_{3} \mathrm{O}_{4}$ nanoparticles in MCF-7 cells when compared with free quercetin. The results showed that the QTSK1 is less toxic compared to the other prepared nanoparticles (Q1-Q4). It is necessary to remember that in this study the purpose is to induce cells death by a photothermal effect. Moreover, the functionnalisation of QTSK1 with glucose (QTSK1@G) and folic acid (QTSK1@G@F) shows better biocompatibility compared to the initial QTSK1. Cell internalization of the synthesized colloids (GQAuNPs@G@F) was checked with a confocal microscope under bright and dark field illumination. For comparison purpose, the glass slide contained regions with identically grown cells which were not exposed to the colloid solution. The images reported in Figures 1 and 4 are from the treated and untreated MIA PaCa-2 cells, the same sample region is seen in bright and dark field conditions. The dark field image (3-D) shows a high density of bright, small scattering centers dispersed all over the glass slide.

The smallest black spots observed bright field may correspond to the aggregates of a size of about $200 \mathrm{~nm}$. Individual nanoparticles are not seen under bright field due to their very small, submicrometric dimensions. Such a background of bright dots is not seen when observing the non-treated cell. Furthermore, the density of these bright spots clearly increases if the original concentration of the incubated colloids is increased. It appears that the colloids have a tendency to accumulate inside the cells: most of them present a central bright region. In the bright field image, dark patches, indicating lower transmission of the incident white light are seen at the corresponding positions for the larger aggregates of nanoparticles.

The internalized metallic nanoparticles may act as nano-sources of heat when irradiated by light of frequency matching their localized surface plasmon resonance. This thermo-plasmonic effect leads to an increase of the local temperature near the irradiated nanoparticle(s) sufficiently high to alter any surrounding biological structures and ultimately provoking cell death. Modification of the cell morphology following absorption of laser light (at $660 \mathrm{~nm}$ ) by the loaded nanoparticles was inspected by comparing microscope images of region before and after laser irradiation. Individual cells were observed with a higher magnification objective (80X) to gain a better insight into the distribution of the colloidal particles. Considering the numerical

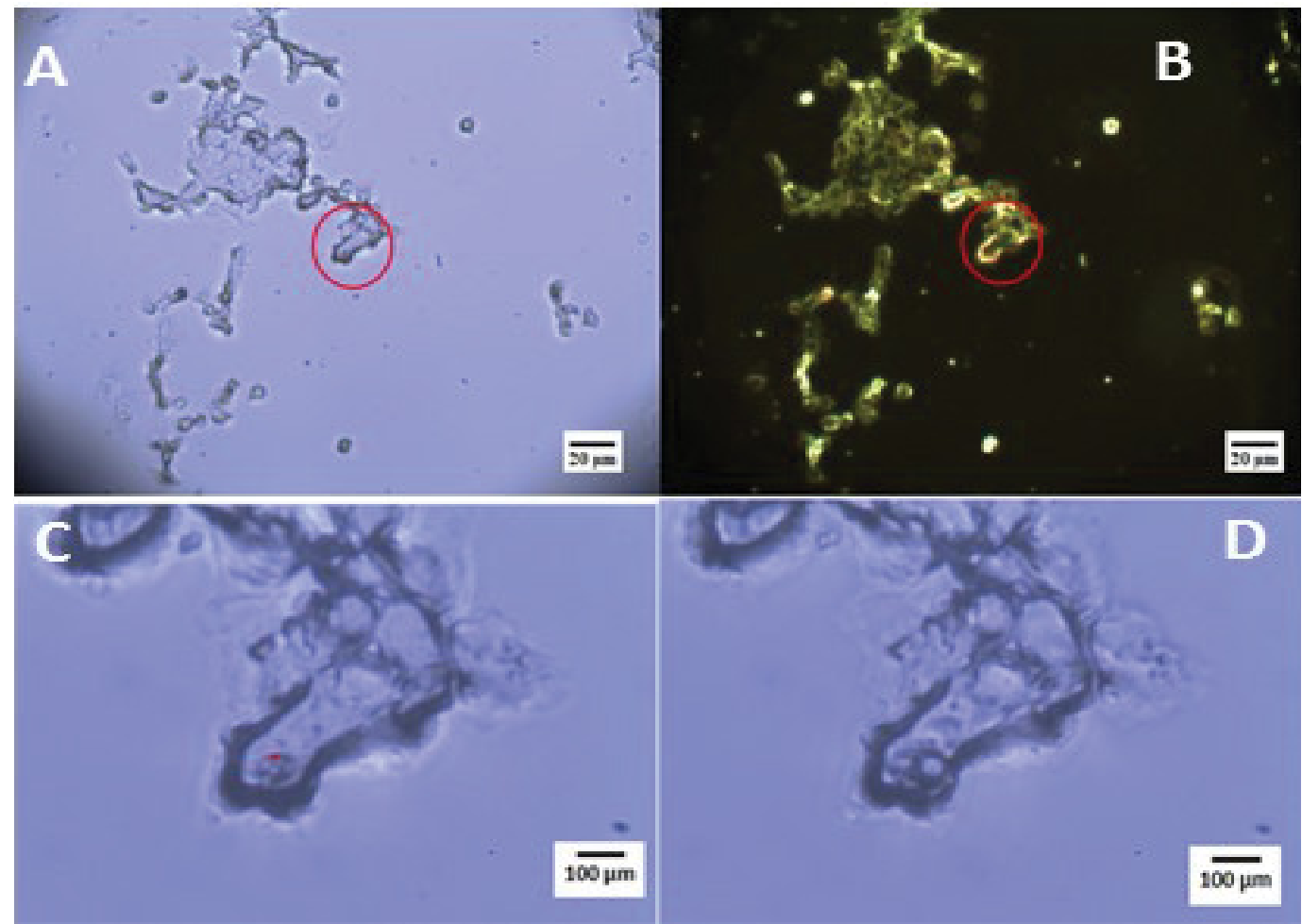

Figure 4: Bright field (A) and dark field (B) images (20x, 0.25 NA objective) of MIA PaCa-2 cells treated with the GQAuNPs@G@F. Bright field (C,D) of the surrounded zone in red at higher magnification images ( $80 \mathrm{x}, 0.75 \mathrm{NA}$ ) of MIAPaCa-2 cells before (left) and after (right) laser irradiation (2.2 $\mathrm{mW} / \mathrm{cm}^{2}$ for $6 \mathrm{~min}$ ). The laser was focused at the position marked with a red dot. 
aperture of the objective used for laser irradiation (80X, $0.75 \mathrm{NA}$ ), the diffraction limited laser spot should have a diameter of roughly one micron.

A first bright field image is recorded with the laser off (4-C). Then the laser is turned on and focused at the desired location onto the cell (red dot). The position of the colloids can be relatively easily guessed by observing the contrast in the bright field image: darker patches indicate the accumulation of the absorbing particles. Once the laser spot $(\mathrm{exc}=660 \mathrm{~nm})$ is at the desired position, laser power is increased (by removing filters) for a fixed time then again turned off. A second optical image is then recorded after switching off the laser light (4D). By comparing the two images, before and after the irradiation, we notice the appearance of a hole due to the cell membrane damage after an exposure of $6 \mathrm{~min}$ and an irradiation of $2.2 \mathrm{~mW} / \mu \mathrm{m}^{2}$. It means that photon energy is converted to heat sufficient to induce cellular damage via thermal effects. Despite that the laser irradiated area is only about one micron in diameter (diameter of the focal spot), the effects of the plasmonic heating extend over a larger portion of the immobilized cell. Negative tests were also carried out: during the focusing of the laser light away from one nanoparticle aggregate, no damage is formed.

The excitation of the localized surface plasmon resonances upon laser irradiation brings also an enhancement of the Raman signal of molecules located in the close proximity of the colloids surface. SERS signal was detected when illuminating the internalized colloid and analyzing the back scattered radiation (Figure 5). A stable Raman signal can be detected from the NP aggregates using $2 \mathrm{~mW} / \mu \mathrm{m}^{2}$ for $3 \mathrm{sec}$. This spectrum is relative to the cell/AuNP system; when focusing away from the colloids but still inside the cell, no Raman signal is detected even for longer acquisition times. The characteristics peaks in the range $500-1200 \mathrm{~cm}^{-1}$ corresponding to amino groups of proteins and others biomolecules in the cells is more evident before irradiation (Figure 5 red line). After laser excitation, we observed a strong disparition of principal peaks corresponding to functional biomolecule in the cell with apparition of amorphous carbon (wide strips $1300-1550 \mathrm{~cm}^{-1}$ ) that confirms the success of photothermal effect inside the MIA PaCa2 cells.

\section{In vivo evaluation of GQAuNPs}

A first in vivo study was performed on a murine melanoma model. This model is a superficial tumor that can be easily irradiated. Indeed even if infrared radiations are used, the depth of penetration is limited to $4-5 \mathrm{~mm}$ in the tissue due to optical diffusion [37]. The mechanism of action of PTT is not dependent on cell type. If the adequate ligand is coated on the AuNPs, this favors the accumulation of GQAuNP and induces hyperthermia when irradiated thus provokes the cell death in various tumor models. As B16F10 melanoma cells overexpressed folate receptors, that was considered as a good candidate to assess GQAuNPs conjugated to folic acid. GQAuNPs coupled with laser irradiation showed a moderate antitumor activity ( $\mathrm{T} / \mathrm{C} \leq 42 \%$ and $\mathrm{rAUC} \leq 50 \%$, Figure 6) and were not toxic at the administrated dose (no weight loss $>20 \%$ ).

An inhibition of tumor growth by $46 \%$ (in all mice and a partial regression in one mouse) were observed. No inhibition of tumor growth was observed in the Laser group indicating that the antitumor effect observed in the NP group is induced by the association of GQAuNPs with laser and not by the laser alone. For a first assay, the results are encouraging even if several parameters need to be improved. First, the irradiation surface, in this study the tumor volume was not fully exposed to the laser but only $40 \%$ of the surface. Second, the delay

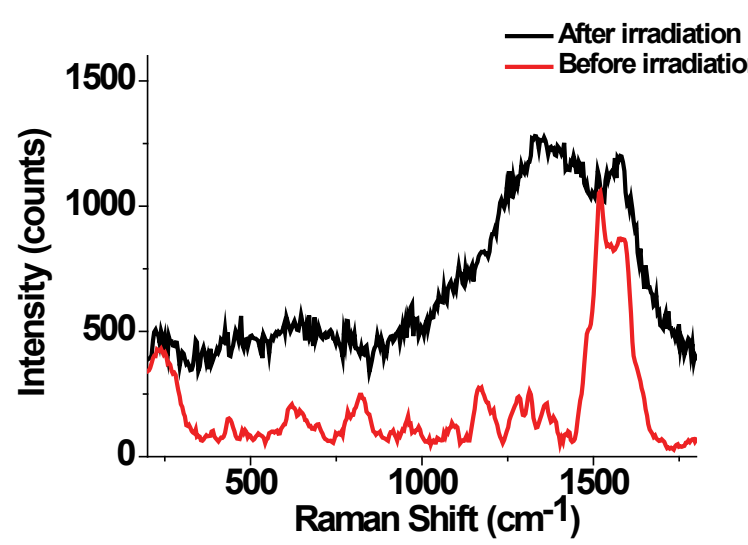

Figure 5: Raman spectra of AuNPs internalized into MIA PaCa-2 cells. The Raman spectra before and after irradiation were acquired with $80 x$ objective, $10 \%$ laser power, 3 s.

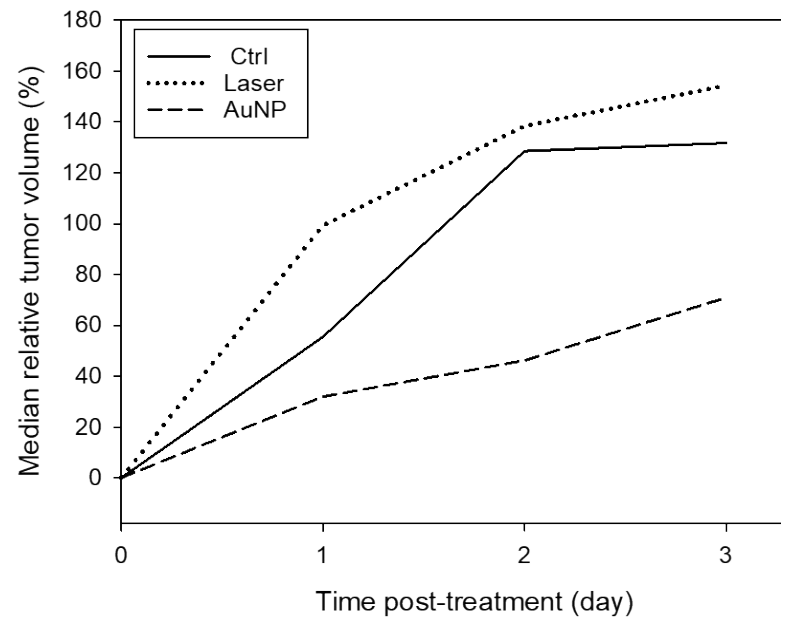

Figure 6: Effects of GQAuNPs administered into the tumor coupled with lase irradiation on the tumor volume of mice bearing subcutaneous B16F10 murine melanoma xenograft.

between injection of GQAuNPs and irradiation in order to have a homogenous distribution of nanoparticles in the tumor volume at the time of irradiation. We are considering photoacoustic imaging studies to determine the biodistribution of the nanoparticles inside the tumor after direct intratumoral delivery. Indeed, due to the optical absorption that results from the SPR effects, gold nanoparticles can be used as photoacoustic contrast agent $[38,39]$. The last parameters to improve are the concentration of GQAuNPs, the duration and the power of the irradiation to get sufficient hyperthermia to induce cell death and so a complete tumor regression. It is important to control the rise of temperature in order to induce cell death by apoptosis process and not by necrosis [40-41]. For that, the temperature have not to exceed $47^{\circ} \mathrm{C}$ and the temperature to reach in vivo is inferior to the temperature we need in vitro due to the microenvironment $[42,43]$. The power density of laser we used was $1.5 \mathrm{~W} / \mathrm{cm}^{2}$, it is less than the majority of power density reported in the literature $\left(2-22 \mathrm{~W} / \mathrm{cm}^{2}\right)$ but the objective is to decrease this value up to $0.2 \mathrm{~W} / \mathrm{cm}^{2}$. This is the maximal permissible exposure of skin as per ANSI regulation for $808 \mathrm{~nm}$ and continuous mode irradiation. Finally, we need to achieve optimal efficiency with a single treatment to avoid inducing adaptation to heat stress 
Citation: Haddada MB, Gerometta E, Bialecki A, Fu W, Zhang Y, et al. (2018) Endemic Plants: From Design to a New Way of Smart Hybrid Nanomaterials for Green Nanomedicine Applications. J Nanomed Nanotechnol 9: 518. doi: 10.4172/2157-7439.1000518

(thermotolerance) due to the expression of heat shock proteins (HSP) [44]. In the case where tumor cells are thermoresistant, it is possible to combine the nanoparticles with HSP inhibitor or small interfering RNA for HSP silencing. More studies are ongoing on two models: melanoma and basal cell carcinoma. Currently, for melanoma and basal cell carcinoma, the main treatment is based on surgical excision whose the risk to involve the formation of permanent scars depends a lot on the localization. Other alternatives are the use of dynamic phototherapy and imiquinod or 5-FU creams. These treatments are limited to superficial basal cell carcinoma, actinic keratosis, Bowen's disease and condyloma for creams. They can present a pain during the application or after the treatment with irritations which can lead to an early stop of their use. With our treatment we can hope to treat these pathologies with less discomfort for patients thanks to a complete regression achieved in one injection.

\section{Conclusion}

This paper reported a green chemistry approach to synthesize AuNPs by the reduction of the $\mathrm{AuCl}_{4}$-ions using Hubertia ambavilla and quercetin. The quercetin is a flavonoid presenting antioxidant activity that is used both as reducing agent and capping agent. The particles morphology formed consists in spherical-shaped nanoparticles depending on the nature of the surfactant as reducing agent. GQAuNPs are highly soluble under physiological conditions and no aggregation was observed even after 3 months storage. The possibility to ablate MIA PaCa- 2 cell line was evaluated but this study confirms the biocompatibility of complex green gold particles and their use as nanoplatform for the thermal destruction of several cancers. A complete destruction of the tumor cells was achieved with a laser power of $2 \mathrm{~mW} / \mu \mathrm{m}^{2}$ for $3 \mathrm{sec}$ at $808 \mathrm{~nm}$. This paper highlights the efficacy of GQAuNPs for the photothermal activity (PTT) of MIA PaCa- 2 cells in order to develop a multitask nanovector with a highly effective cancer therapy. The first in vivo study confirms the efficacy of GQAuNPs for the PTT and even if some parameters need to be optimized this treatment looks promising.

\section{References}

1. Wang Q, Zhuang X, Mu J, Deng ZB, Jiang H, et al. (2013) Delivery of therapeutic agents by nanoparticles made of grapefruit-derived lipids. Nat Commun 4: 1867.

2. Hu Q, Li H, Wang L, Gu H, Fan C (2018) DNA Nanotechnology-Enabled Drug Delivery Systems. Chem Rev.

3. Sukhanova A, Bozrova S, Sokolov P, Berestovoy M, Karaulov A, et al (2018) Dependence of Nanoparticle Toxicity on Their Physical and Chemical Properties. Nanoscale Res Lett 13: 44.

4. Reid BT, Reed SM (2016) Improved methods for evaluating the environmenta impact of nanoparticle synthesis. Green Chemistry 18: 4263-4269.

5. Bahadar H, Maqbool F, Niaz K, Abdollahi M (2016) Toxicity of nanoparticles and an overview of current experimental models. Iran Biomed J 20: 1-11.

6. Khan I, Saeed K, Khan I (2017) Nanoparticles: Properties, applications and toxicities. Arab J Chem.

7. Gunduz N, Ceylan H, Guler MO, Tekinay AB (2017) Intracellular accumulation of gold nanoparticles leads to inhibition of macropinocytosis to reduce the endoplasmic reticulum stress. Scientific Reports 7: 40493.

8. Fadeel B, Fornara A, Toprak MS, Bhattacharya K (2015) Keeping it real: The importance of material characterization in nanotoxicology. Biochem Biophys Res Commun 468: 498-503.

9. Spadavecchia J, Apchain E, Albéric M, Fontan E, Reiche I (2014) One $\square$ Step Synthesis of Collagen Hybrid Gold Nanoparticles and Formation on Egyptian like Gold $\square$ Plated Archaeological Ivory. Angew Chem Int Ed Engl 53: 83638366.
10. He Z, Liu J, Du L (2014) The unexpected effect of PEGylated gold nanoparticles on the primary function of erythrocytes. Nanoscale 6: 9017-9024.

11. Kreyling WG, Abdelmonem AM, Ali Z, Alves F, Geiser M, et al. (2015) In vivo integrity of polymer-coated gold nanoparticles. Nature Nanotechnology 10: 619

12. Murthy SK (2007) Nanoparticles in modern medicine: state of the art and future challenges. Inter J Nanomed 2: 129-141.

13. Alexandridis $P$, Tsianou M (2011) Block copolymer-directed metal nanoparticle morphogenesis and organization. Eur Polymer J 47: 569-583.

14. Monteil M, Moustaoui H, Picardi G, Aouidat F, Djaker N, et al. (2018) Polyphosphonate ligands: From synthesis to design of hybrid PEGylated nanoparticles toward phototherapy studies. J Colloid Interface Sci 513: 205213.

15. Nakao A, Fujiki M (2015) Visualizing spontaneous physisorption of noncharged $\pi$-conjugated polymers onto neutral surfaces of spherical silica in nonpolar solvents. Poly J 47: 434.

16. Heinz H, Pramanik C, Heinz O, Ding Y, Mishra RK, et al. (2017) Nanoparticle decoration with surfactants: molecular interactions, assembly, and applications. Surface Science Reports 72: 1-58.

17. Morel AL, Giraud S, Bialecki A, Moustaoui H, de La Chapelle ML, et al. (2017) Green extraction of endemic plants to synthesize gold nanoparticles for theranostic applications. Frontiers in Laboratory Medicine 1: 158-171.

18. Politi LDSJ, Longobardi S, Giardina P, Rea I, Methivier C, et al. (2015) Extinction coefficient of gold nanoparticles with different sizes and different capping ligands. Colloids and Interfaces B 136: 214-121.

19. Heck JG, Napp J, Simonato S, Möllmer J, Lange M, et al. (2015). Multifunctional Phosphate-Based Inorganic-Organic Hybrid Nanoparticles. J Am Chem Soc 137: 7329-7336.

20. Shi Y, Liu M, Deng F, Zeng G, Wan Q, et al. (2017) Recent progress and development on polymeric nanomaterials for photothermal therapy: a brief overview. J Mat Che B 5: 194-206.

21. Huang X, El-Sayed MA (2011) Plasmonic photo-thermal therapy (PPTT) Alexandria J Med 47

22. Chen C, Wang S, Li L, Wang P, Chen C, et al. (2016) Bacterial magnetic nanoparticles for photothermal therapy of cancer under the guidance of MRI. Biomaterials 104: 352-360.

23. Das DK, Chakraborty A, Bhattacharjee S, Dey S (2013) Biosynthesis of stabilised gold nanoparticle using an aglycone flavonoid, quercetin. J Experimental Nanoscience 8: 649-655.

24. Abdelhalim MAK, Moussa SAA, Qaid HAY (2018) The protective role of quercetin and arginine on gold nanoparticles induced hepatotoxicity in rats. Inter J Nanomed 13: 2821

25. Bishayee K, Khuda-Bukhsh AR, Huh SO (2015) PLGA-loaded goldnanoparticles precipitated with quercetin downregulate HDAC-Akt activities controlling proliferation and activate p53-ROS crosstalk to induce apoptosis in hepatocarcinoma cells. Mol Cells 38: 518-527.

26. Murillo E, Britton GB, Durant AA (2012) Antioxidant activity and polypheno content in cultivated and wild edible fruits grown in Panama. J Pharm Bioallied Sci 4: 313-317.

27. Ganesan K, Xu B (2017) A critical review on polyphenols and health benefits of black soybeans. Nutrients 9: 455

28. Deokar GK, Ingale AG (2016) Green synthesis of gold nanoparticles (Elixir of Life) from banana fruit waste extract-an efficient multifunctional agent. RSC Advances 6: 74620-74629.

29. Liu H, Jiang P, Li Z, Li X, Djaker N, et al. (2018) HIV $\square 1$ Tat Peptide $\square$ Gemcitabine Gold (III) $\square$ PEGylated Complex-Nanoflowers: A Sleek Thermosensitive Hybrid Nanocarrier as Prospective Anticancer. Particle \& Particle Systems Characterization.

30. Moustaoui H, Movia D, Dupont N, Bouchemal N, Casale S, et al. (2016) Tunable design of gold (III)-doxorubicin complex-pegylated nanocarrier. the golden doxorubicin for oncological applications. ACS Appl Mater Interfaces 8 : 19946-19957.

31. Marguerit G, Moustaoui H, Haddada MB, Djaker N, de la Chapelle ML, et al. (2018) Taxanes Hybrid Nanovectors: From Design to Physico $\square$ Chemical Evaluation of Docetaxel and Paclitaxel Gold (III) $\square$ PEGylated Complex Nanocarriers. Particle \& Particle Systems Characterization 35: 1700299. 
Citation: Haddada MB, Gerometta E, Bialecki A, Fu W, Zhang Y, et al. (2018) Endemic Plants: From Design to a New Way of Smart Hybrid Nanomaterials for Green Nanomedicine Applications. J Nanomed Nanotechnol 9: 518. doi: 10.4172/2157-7439.1000518

32. Dario MF, Oliveira CA, Cordeiro LR, Rosado C, Inês de Fátima AM, et al. (2016) Stability and safety of quercetin-loaded cationic nanoemulsion: In vitro and in vivo assessments. Coll Surf A: Physicochem Eng Asp 506: 591-599.

33. Wang J, Zhou N, Zhu Z, Huang J, Li G (2007) Detection of flavonoids and assay for their antioxidant activity based on enlargement of gold nanoparticles. Anal Bioanal Chem 388: 1199-1205

34. Choi Y, Choi MJ, Cha SH, Kim YS, Cho S, et al. (2014) Glucomannan-mediated facile synthesis of gold nanoparticles for catalytic reduction of 4-nitrophenol. Nano Res Lett 9: 103-103.

35. I Capek (2017) Formulation, stabilisation and encapsulation of bacteriophage for phage therapy. Adv Colloid Interface Sci.

36. Vyas A, Dandawate P, Padhye S, Ahmad A, Sarkar F (2013) Perspectives on new synthetic curcumin analogs and their potential anticancer properties. Curr Pharm Des 19: 2047-2069.

37. Choi HS, Liu W, Misra P, Tanaka E, Zimmer JP, et al. (2007) Renal clearance of quantum dots. Nature Biotech 25: 1165.

38. Jurasekova Z, Garcia $\square$ Ramos JV, Domingo C, Sanchez $\square$ Cortes S (2006) Surface enhanced Raman scattering of flavonoids. J Raman Spectro 37: 1239-1241.
39. HWu HY, Huang YT, Shen PT, Lee H, Oketani R, et al. (2016) Ultrasmall all-optical plasmonic switch and its application to superresolution imaging. Scientific Reports 6: 24293.

40. Huang X, El-Sayed IH, Qian W, El-Sayed MA (2006) Cancer cell imaging and photothermal therapy in the near-infrared region by using gold nanorods. J Am Chem Soc 128: 2115-2120.

41. Brigger I, Dubernet C, Couvreur P (2012) Nanoparticles in cancer therapy and diagnosis. Adv Drug Deliv Rev 64: 24-36.

42. Boisselier E, Astruc D (2009) Gold nanoparticles in nanomedicine: preparations, imaging, diagnostics, therapies and toxicity. Chem Soc Rev 38: 1759-1782.

43. Sabale S, Kandesar P, Jadhav V, Komorek R, Motkuri RK, et al. (2017) Recent developments in the synthesis, properties, and biomedical applications of core/ shell superparamagnetic iron oxide nanoparticles with gold. Biomater Sci 5 : 2212-2225.

44. Kumar SR, Priyatharshni S, Babu VN, Mangalaraj D, Viswanathan C, et al. (2014) Quercetin conjugated superparamagnetic magnetite nanoparticles for in-vitro analysis of breast cancer cell lines for chemotherapy applications. J Colloid Interface Sci 436: 234-242. 